\title{
Design of a Nonlinear Dynamic Inversion Controller for Trajectory Following and Maneuvering for Fixed Wing Aircraft
}

\author{
Abhay A. Pashilkar, Shaik Ismail \\ Scientists, Flight Mechanics and Control Division \\ National Aerospace Laboratories \\ Bangalore - 560017, India \\ apash@nal.res.in,shaik1752@gmail.com
}

\author{
Ramakalyan Ayyagari \\ Prof., Dept. of Instrumentation and Control Engg., \\ National Institute of Technology \\ Tiruchirappalli, India \\ rkalyn@gmail.com
}

\author{
Narasimhan Sundararajan \\ Prof. (retd), School of Electrical \& Electronic Engineering \\ Nanyang Technological University, Singapore 639798 \\ ensundara@ntu.edu.sg
}

\begin{abstract}
This paper presents the design of a robust linear controller that can be used for trajectory following and maneuvering of fixed-wing aircraft using Nonlinear Dynamic Inversion (NDI) principles. The design addresses control coupling to exploit multiple redundant controls. It can also be easily extended to state decoupling. The design procedure exploits the nature of the equations of motion written in the wind axis resulting in a cascaded linear controller structure with inner and outer loops. A systematic methodology is evolved which uses only the relevant stability and control derivatives in the control synthesis, as opposed to the inversion of the complete nonlinear equations used in conventional NDI designs. The tuning of the control gains is based on the requirements of adequate trajectory following and robustness to control surface failures. Finally, it is shown how a series of controllers can be derived depending on the sensor complement available on the aircraft. The proposed approach is ideal for fixed-wing Unmanned Aerial Vehicles (UAVs).
\end{abstract}

Keywords-Nonlinear Dynamic Inversion, , fixed-wing aircraft, trajectory following, unmanned ariel vehicle

\section{INTRODUCTION}

Flight control law design methods based on NDI have evolved immensely due to advances in both hardware and theoretical developments over the past decades [1-3, 6-8]. These developments have culminated in the flight testing of NDI based control laws [9].

Early attempts to apply feedback linearization theory or NDI techniques to flight control and trajectory following began appearing in the mid and late 1980's [1-3]. Reference [1] provides the basic theory for feedback linearization for the aircraft equations of motion when cast in the block triangular form. Lane and Stengel [2] explained that the aerodynamic control surfaces must be treated primarily as moment generators and their force contributions must be neglected. Menon et al. [3], made the time-scale separation concept explicit for trajectory following controllers. It is now known that the NDI controller is not robust to parameter perturbations as it is based on cancellation of nonlinearities in the plant dynamics.

In the early 1990's the idea of integrator backstepping was developed by Kokotovic and co-workers [4]. The block triangular nature of the aircraft equations of motion allows us to apply backstepping for flight control. Backstepping improves the robustness of NDI significantly by introducing a feedback element into the control structure. Due to this positive attribute, backstepping was applied to the problem of flight control [5-7]. In these papers we find a clear demarcation of the states involved in the block triangular form of the equations of motion along with the explicit intent to design the flight control system based on multiple time scale separation principles. Snell et al. [6] also showed how multiple redundant control surfaces could be handled in the NDI framework using the matrix pseudo-inverse. The use of stability axis rates as opposed to body axis in the innermost loops as a natural choice for flight control is clearly seen in references [7-8]. In addition, the latter [8] explains the various methods of control allocation available to the designer - namely explicit ganging, pseudo control, pseudo inverse and daisy chaining. This reference also explains the process of designing feed forward loops to improve the tracking performance of the dynamic inversion controller. Harkegard [10] has shown that it is not necessary to cancel all nonlinearities in the equations of dynamics. In fact, in many cases it is possible to retain the useful nonlinearities and thereby reduce control effort. Further in many cases a sufficiently high linear negative feedback gain can make the adaptive backstepping controller globally asymptotically stable. Later Van Oort [11] extended the results to non-affine systems. In passing we also mention a variation of NDI called 
Incremental Nonlinear Dynamic Inversion [12] where the angular acceleration is predicted from inertial measurements of the angular rates and used in the innermost NDI loop.

Control surface allocation amongst the multiple redundant controls available in modern fixed wing aircraft is a recurring issue which has been addressed in $[6,8,10,13]$.

In this paper we apply the NDI concepts to develop a linear controller. Use of the wind axis coordinates particularly the wind axis roll and yaw rates simplify the controller sufficiently permitting us to neglect the nonlinear terms while retaining stability. We also demonstrate how to select the gains to be used in the various loops whist maintaining dynamic separation between the inner and outer loops. The authors believe that this approach is sufficiently generic to allow for application to the class of fixed wing aircraft.

The linear controller is motivated by the fact that sufficiently high linear gains can make the system globally asymptotically stable even if we do not cancel some of the nonlinearities [10]. Linear analysis tools are well developed and traditional gain and phase margins can be easily provided for certification [8]. With integrator backstepping, direct method of changing the loop bandwidth is available to prevent control surface / rate saturations $[5,8]$. The added advantage of using NDI concepts is the ability to add nonlinear correction terms to improve the state decoupling (e.g., angular velocity cross product in the moment equations to handle inertia coupling). Finally, many UAV's fly at a fixed flight condition and therefore a single point linear design is sufficient.

The paper is organized as follows. Section II describes the aircraft model and actuator models used in the paper. It also contains the equations of motion based on which the NDI control structure is evolved. The linear model data which forms the basis for innermost loop design is also provided in this Section. In Section III the NDI controller is developed loop by loop proceeding from the innermost rotational equations loop design to the outermost trajectory following loop. The sixdegree-of-freedom simulation results are presented in Section IV.

\section{AIRCRAFT CHARACTERISTICS}

\section{A. Aircraft Dynamics}

The aircraft model used in this study is that of a high performance fighter aircraft. Details of the model can be found in [14]. For the purpose of this study, the elevator and aileron control surface aerodynamic data has been split into two parts corresponding to left and right surface using CFD computations [15]. The aerodynamic model also contains a ground effect model. The aircraft has two elevators $(-25$ to +25 deg deflections), which can be moved together or in differential mode. It also has a pair of ailerons ( -20 to +20 deg deflection), and a rudder $(-30$ to +30 deg deflection). The engine model (without dynamics) completes the six-degree of freedom simulation model.

The aircraft has hydraulic actuators, which drive the primary control surfaces that are modeled as first order lags with a time constant of $50 \mathrm{msec}$. The rate limits for the actuators is set at $60 \mathrm{deg} / \mathrm{s}$. The entire mathematical model of the aircraft and controllers was implemented in Simulink/ Matlab.

Standard aeronautical symbols have been used for all the variables in this paper.

\section{B. Equations of Motion}

Under the rigid body assumption, the equations of motion have been taken from [16]. The roll rate and yaw rate have been transformed into stability axes for the purpose of developing the control laws based on dynamic inversion.

\section{Rotational Equations:}

$\left[\begin{array}{c}\dot{p} \\ \dot{q} \\ \dot{r}\end{array}\right]=I^{-1}\left\{-\left[\begin{array}{ccc}0 & -r & q \\ r & 0 & -p \\ -q & p & 0\end{array}\right] I\left[\begin{array}{l}p \\ q \\ r\end{array}\right]+\left[\begin{array}{l}l_{a} \\ m_{a} \\ n_{a}\end{array}\right]+\left[\begin{array}{l}l_{T} \\ m_{T} \\ n_{T}\end{array}\right]\right\}$

Wind Axis Equations:

$$
\begin{aligned}
& \dot{\mu}=p_{s} \cos \beta+(q-\dot{\alpha}) \sin \beta+\dot{\chi} \sin \gamma \\
& \dot{\alpha}=q-p_{s} \tan \beta-\frac{(L-m g \cos \mu \cos \gamma)}{m V \cos \beta} \\
& \dot{\beta}=-r_{s}+\frac{g}{V} \sin \mu \cos \gamma-\frac{Q}{m V}
\end{aligned}
$$

Velocity Vector Equations:

$$
\begin{aligned}
& \dot{V}=\frac{(T \cos \varepsilon \cos v-D)}{m}-g \sin \gamma \\
& \dot{\gamma}=\frac{T(\sin \varepsilon \cos \mu-\cos \varepsilon \sin v \sin \mu)}{m V}+\frac{Q \sin \mu}{m V}+ \\
& \frac{(L \cos \mu-m g \cos \gamma)}{m V} \\
& \dot{\chi}=\frac{L \sin \mu}{m V \cos \gamma}+\frac{T(\cos \varepsilon \sin V \cos \mu+\sin \varepsilon \sin \mu)}{m V \cos \gamma} \\
& -\frac{Q \cos \mu}{m V \cos \gamma}
\end{aligned}
$$

Navigational Equations:

$$
\begin{aligned}
& \dot{x}=V \cos \gamma \cos \chi \\
& \dot{y}=V \cos \gamma \sin \chi \\
& \dot{h}=V \sin \gamma
\end{aligned}
$$

where,

$$
\left[\begin{array}{c}
p_{s} \\
r_{s}
\end{array}\right]=\left[\begin{array}{cc}
\cos \alpha & \sin \alpha \\
-\sin \alpha & \cos \alpha
\end{array}\right]\left[\begin{array}{l}
p \\
r
\end{array}\right]=T\left[\begin{array}{l}
p \\
r
\end{array}\right]
$$

is the body to stability axis transformation. 


\section{Linear Aircraft Model}

A linear model of the aircraft is required for controller design. The nonlinear model of the aircraft was trimmed at the straight and level flight condition ( $\mathrm{Vel}=82.66 \mathrm{~m} / \mathrm{s}$, and $\mathrm{Alt}=600$ $\mathrm{m})$.

The straight and level flight condition consists of the following equilibrium values of the state variables: $u=81.31 \mathrm{~m} / \mathrm{s}, \quad v=0 \mathrm{~m} / \mathrm{s}, \quad w=16.08 \mathrm{~m} / \mathrm{s}, \quad p=0 \mathrm{rad}$, $q=0 \mathrm{rad}, r=0 \mathrm{rad}, \phi=0 \mathrm{rad}, \theta=11.19 \mathrm{deg}, \psi=0 \mathrm{rad}$, $h=600 m$, and $y=0 m$.

Similarly, the corresponding values of the control deflections are: $\delta_{e}=-0.64 \mathrm{deg}, \delta_{a}=0 \mathrm{deg}, \delta_{r}=0 \mathrm{deg}$, and $\delta_{t h r}=0.22$.

In the design process in the following sections, we use the linearized model of the high performance fighter aircraft, at the straight level flight condition only for the innermost loop (rotational equations of motion). The inner loop states are $x=\left[\begin{array}{lll}q & p & r\end{array}\right]^{T}$. The right hand sides of the equations of rotational motion have a dependency on the rotational rates as well as the angle of attack, sideslip and the control surface deflections. The linearized equations can be written as:

$\dot{x}=A y+B u$

where,

$$
\begin{aligned}
& y=\left[\begin{array}{lllll}
\alpha & q & \beta & p & r
\end{array}\right]^{T} \\
& u=\left[\begin{array}{lllll}
\delta_{e-l e f t} & \delta_{e-r i g h t} & \delta_{a-l e f t} & \delta_{a-r i g h t} & \delta_{r}
\end{array}\right]^{T}
\end{aligned}
$$

The angles and rates in the state equations are in radians and $\mathrm{rad} / \mathrm{s}$ respectively, while the control surface deflections are in degrees. The values for the matrices A and B are as follows:

$$
\begin{aligned}
A & =\left[\begin{array}{lrrrr}
0.8145 & -0.6491 & -0.0066 & 0 & -0.0029 \\
0 & 0.0003 & -14.1548 & -1.7533 & 0.8792 \\
0 & 0.0025 & 1.8847 & -0.0482 & -0.2424
\end{array}\right] \\
B & =\left[\begin{array}{rrrrr}
-0.0299 & -0.0299 & 0.0005 & 0.0005 & 0 \\
0.0549 & -0.0549 & 0.0842 & -0.0842 & 0.0340 \\
0.0074 & -0.0074 & 0.0007 & -0.0007 & -0.0169
\end{array}\right]
\end{aligned}
$$

\section{NONLINEAR DYNAMIC INVERSION CONTROLLER DESIGN}

The design begins by considering the three rotational axes for feedback control.

\section{A. Inner Loop Control Decoupling}

We proceed to decouple the control surfaces which appear in the rotational equations by applying dynamic inversion to the linearized equations of motion.

It is noted that we have expressed the rotational dynamics as a function of the states as well as individual control surface deflections. We propose three pseudo controls $\bar{u}=\left[\begin{array}{lll}\delta_{\text {pitch }} & \delta_{\text {roll }} & \delta_{\text {yaw }}\end{array}\right]^{T}$

to exercise decoupled control of each of the three rotational axes. We also transform the roll and yaw rates into the stability axes. Thus, the transformed state vector $\bar{x}, \bar{y}$ are given by:

$$
\begin{aligned}
& \bar{x}=\left[\begin{array}{lll}
q & p_{s} & r_{s}
\end{array}\right]^{T} \\
& \bar{y}=\left[\begin{array}{lllll}
\alpha & q & \beta & p_{s} & r_{s}
\end{array}\right]^{T}
\end{aligned}
$$

The transformation matrices between the original variables $x, y, u$ and their corresponding transformed quantities $\bar{x}, \bar{y}, \bar{u}$ are respectively given by:

$$
\begin{aligned}
x & =\left[\begin{array}{c|c}
1 & 0_{1 \times 2} \\
\hline 0_{2 \times 1} & T^{-1}
\end{array}\right] \bar{x}=T_{1} \bar{x} \\
y & =\left[\begin{array}{c|c}
I_{3 \times 3} & 0_{3 \times 2} \\
\hline 0_{2 \times 3} & T^{-1}
\end{array}\right] \bar{y}=T_{2} \bar{y} \\
u & =\left[\begin{array}{ccc}
1 & -\mathrm{K}_{\mathrm{aei}} & -\mathrm{K}_{\mathrm{rei}} \\
1 & \mathrm{~K}_{\mathrm{aei}} & \mathrm{K}_{\mathrm{rei}} \\
0 & -1 & 0 \\
0 & 1 & 0 \\
0 & \mathrm{~K}_{\text {ari }} & 1
\end{array}\right] \bar{u}=S \bar{u}
\end{aligned}
$$

In the above equations for transformation of the control matrix, the gain $\mathrm{K}_{\text {ari }}$ is the well known ailerons to rudder interconnect. This is routinely used in traditional flight control design for suppressing the development of sideslip due to deflection of aileron by application of proportional rudder.

We have introduced two additional gains $\mathrm{K}_{\text {aei }}$ and $\mathrm{K}_{\text {rei }}$ which are intended to exploit the capability of the elevators in differential mode to generate additional rolling moments and yawing moments respectively. While the former gain enables the controller to handle a new type of failure (namely failure of both ailerons), the latter gain allows the controller to handle a larger range of rudder failures.

With the above transformations, the linearized equations for rotational dynamics can be written as:

$$
T_{1} \dot{\bar{x}}=T_{2} \bar{y}+B S \bar{u}
$$

These equations can be rearranged as:

$$
(B S)^{+} T_{1} \dot{\bar{x}}=(B S)^{+} T_{2} \bar{y}+\bar{u}
$$

where $(B S)^{+}$is the matrix pseudo-inverse of the matrix product $B S$.

The gains $\mathrm{K}_{\text {ari }}, \mathrm{K}_{\text {aei }}$ and $\mathrm{K}_{\text {rei }}$ in the matrix $S$ are chosen such that $(B S)^{+} T_{1}$ is a diagonal matrix resulting in the control decoupling of the pitch, roll and yaw axes. Application of this to our aircraft model with $\mathrm{K}_{\mathrm{ari}}=1.66 \mathrm{deg} / \mathrm{deg}, \mathrm{K}_{\mathrm{aei}}=0.75 \mathrm{deg} / \mathrm{deg}$ 
and $\mathrm{K}_{\mathrm{rei}}=0.27 \mathrm{deg} / \mathrm{deg}$ results in the following decoupled equations:

$$
\begin{aligned}
& -16.7 \dot{q}=-13.6 \alpha+10.9 q+\delta_{\text {pitch }} \\
& -5 \dot{p}_{s}=67.9 \beta+7.7 p_{s}-5.8 r_{s}+\delta_{\text {roll }} \\
& -46.8 \dot{r}_{s}=-222 \beta-10.3 p_{s}+22.1 r_{s}+\delta_{\text {yaw }}
\end{aligned}
$$

Each of the above equations states the dependence of the rotational rate on the relevant aircraft states and the control input. The states $\alpha$ and $\beta$ are in radians, while the rates are in $\mathrm{rad} / \mathrm{sec}$. Dynamic inversion control is designed to create a first order response in the variable being controlled. For example, we choose the pitch command to be:

$$
\delta_{\text {pitch }}=+13.6 \alpha-10.9 q+K_{q}\left(q_{c m d}-q\right)
$$

where it is noted that $q_{c m}$ is the commanded pitch rate input. It is noted that this "control law" amounts to both control decoupling as well as state decoupling in the longitudinal equations.

Substituting this "control law" into the first equation (25) for pitch acceleration results in the desired first order response of the aircraft pitch rate to a step input pitch rate command:

$\dot{q}=\frac{K_{q}}{-16.7}\left(q_{c m d}-q\right)$

By choosing a value of $\mathrm{K}_{\mathrm{q}}=-105 \mathrm{deg} / \mathrm{rad} / \mathrm{sec}$ for the proportional pitch rate feedback gain, the effective time constant of the first order response works out to be:

$\tau=\frac{-16.7}{-105}=0.16 s$

The value of $\mathrm{K}_{\mathrm{q}}$ is chosen to ensure that the actuators do not rate limit during the landing manoeuvre. The pitch control law equation can be further simplified by noting that the $\mathrm{K}_{\mathrm{q}}$ gain is very high compared to the first two terms. The simplification amounts to only control decoupling, while state coupling in longitudinal axis from the lateral-directional axes is seen to be negligible. Therefore, we finally have:

$$
\delta_{\text {pitch }} \cong K_{q}\left(q_{\text {cmd }}-q\right)
$$

In a similar manner, we have the roll and yaw axis inner loop control laws as:

$$
\begin{aligned}
& \delta_{\text {roll }} \cong K_{p_{s}}\left(p_{\text {scmd }}-p_{s}\right) \\
& \delta_{\text {yaw }} \cong K_{r_{s}}\left(r_{s c m d}-r_{s}\right)
\end{aligned}
$$

It is noted that although the coefficients multiplying $\beta$ in the roll and yaw equations are high, when we consider small values for sideslip and convert to degrees, these terms can be neglected in the dynamics. The value of the roll and yaw gains is $\mathrm{K}_{\mathrm{ps}}=-25 \mathrm{deg} / \mathrm{rad} / \mathrm{s}$ and $\mathrm{K}_{\mathrm{rs}}=-180 \mathrm{deg} / \mathrm{rad} / \mathrm{s}$ resulting in the first order response time constants of $0.2 \mathrm{~s}$ and $0.26 \mathrm{~s}$ respectively.
A final point to be noted before proceeding to the design of the other loops is that the angle of attack figures in the transformation of the body axis roll and yaw rates to the stability axis roll and yaw rates. If the measured value of the angle of attack is used for this transformation, this creates a coupling between the longitudinal and lateral-directional axes. This is avoided by using the nominal $1-\mathrm{g}$ angle of attack for this transformation.

\section{B. Outer Loop Control Design $(\mu, \alpha, \beta)$}

In the first approximation, we assume that the flight path angle $(\gamma)$, angle about velocity vector $(\mu)$ and sideslip $(\beta)$ are small, lift balances the weight and the side force $Q$ is negligible. This results in the following dynamics:

$\dot{\alpha} \cong q$

$\dot{\mu} \cong p_{s}$

$\dot{\beta} \cong-r_{s}+\frac{g}{V} \cdot \mu$

The outer loop "control law" based on the above equations is:

$$
\begin{aligned}
& q_{c m d}=K_{a l p h a}\left(\alpha_{c m d}-\alpha\right) \\
& p_{s c m d}=K_{m u}\left(\mu_{c m d}-\mu\right) \\
& r_{c m d}=-K_{\text {beta }}\left(\beta_{c m d}-\beta\right)+\frac{g}{V} \cdot \mu
\end{aligned}
$$

The gains $\mathrm{K}_{\text {alpha }}=2.5 \mathrm{rad} / \mathrm{s} / \mathrm{rad}, \mathrm{K}_{\mathrm{mu}}=3.0 \mathrm{rad} / \mathrm{s} / \mathrm{rad}$ and $\mathrm{K}_{\text {beta }}=1.0 \mathrm{rad} / \mathrm{s} / \mathrm{rad}$ are used resulting in the first order time constants $0.4 \mathrm{~s}, 0.33 \mathrm{~s}$ and $1.0 \mathrm{~s}$ respectively. It is noted that the outer loop time constants are at least 2.5 times of the inner loop time constants ensuring a significant dynamic separation between the cascaded loops.

\section{Tracking Loop Control Design $(V, \gamma, \chi)$}

Similarly if we assume that the angles $\varepsilon, \nu, \gamma, \mu$ are small and the sideforce $Q$ is negligible (i.e., turns are coordinated), in the first approximation the velocity vector equations are:

$$
\begin{aligned}
& \dot{V} \cong \frac{(T-D)}{m}-g \cdot \gamma \approx b_{v t h r} \cdot \delta_{t h r}-g \cdot \gamma \\
& \dot{\gamma} \cong \frac{(L-m g)}{m V} \approx \frac{\bar{q} S C_{L \alpha}}{m V}\left(\alpha-\alpha_{t r i m}\right) \\
& \dot{\chi} \cong \frac{L \cdot \mu}{m V} \approx \frac{g}{V} \mu
\end{aligned}
$$

In the above equations $b_{v t h r}$ is the gradient of aircraft acceleration equation with respect to throttle deflection. This term typically appears in the $B$ matrix. 
$b_{v t h r}=\frac{\partial \dot{V}}{\partial \delta_{t h r}}$

The $C_{L \alpha}$ is the gradient of the lift curve slope and $\alpha_{\text {trim }}$ is the trim angle of attack. The dynamic pressure is represented by $\bar{q}$. Equation (41) is conveying that for a fixed wing aircraft the curvature of the flight path in the vertical plane is achieved by increasing the lift force through changing the angle-of-attack. Similarly, equation (42) indicates that the flight path curvature in the horizontal plane is achieved by tilting the lift vector in the direction of the turn. The "control law" for tracking loop is then developed based on the above equations:

$$
\begin{aligned}
& \delta_{t h r}=\frac{1}{b_{v t h r}}\left[K_{v e l}\left(V_{c m d}-V\right)+g \cdot \gamma\right] \\
& \alpha_{c m d}=\frac{m V}{\bar{q} S C_{L \alpha}} K_{g a m}\left(\gamma_{c m d}-\gamma\right)+\alpha_{t r i m} \\
& \mu_{c m d}=\frac{V}{g} K_{c h i}\left(\chi_{c m d}-\chi\right)
\end{aligned}
$$

The gains are $\mathrm{K}_{\mathrm{vel}}=0.5 \mathrm{~s}^{-1}, \mathrm{~K}_{\mathrm{gam}}=1.0 \mathrm{rad} / \mathrm{sec} / \mathrm{rad}$ and $\mathrm{K}_{\mathrm{chi}}=$ $0.5 \mathrm{~s}^{-1}$. These values result in first order time constants of $2 \mathrm{~s}$, $1 \mathrm{~s}$ and $2 \mathrm{~s}$ respectively for these loops. The time constants of the flight path and the heading angle loops is at least 2.5 times those of the angle of attack and bank angle loops thereby ensuring dynamic separation.

\section{Position Loop Control Design $(y, h)$}

The equations for position in the first approximation are:

$$
\dot{y} \cong-V \cdot\left(\chi_{c m d}-\chi\right)
$$

$\dot{h} \cong V \cdot \gamma$

The "control law" for position loop is then developed based on the above equations:

$$
\begin{aligned}
& \left(\chi_{c m d}-\chi\right)=\frac{-1}{V} K_{y}\left(y_{c m d}-y\right) \\
& \gamma_{c m d}=\frac{1}{V} K_{h}\left(h_{c m d}-h\right)
\end{aligned}
$$

The gains are $\mathrm{K}_{\mathrm{y}}=0.5 \mathrm{rad} / \mathrm{s}$ and $\mathrm{K}_{\mathrm{h}}=0.55 \mathrm{rad} / \mathrm{s}$. These values result in first order time constants of $2 \mathrm{sec}$ and $1.82 \mathrm{sec}$ respectively for these loops. The time constants of the cross track and the altitude loops is at least 1.82 times those of the bank angle and flight path angle loops thereby ensuring dynamic separation.

It is desirable to enhance the ramp following of the control system to altitude and track angle. This is achieved by adding a feedforward signal proportional to the derivative of the altitude and track angle commands respectively. The derivative is constructed using a washout filter.
The longitudinal and lateral-directional closed loop scheme is shown in Figs. 2 and 3. It is noted that we have added Washout filters (denoted as 'WO') and PID blocks (denoted as 'PID') in these figures. The airspeed loop also has an additional lead-lag compensator to improve the speed of response without compromising the overshoot.

\section{E. Alternate Feedback Signals}

The use of angle of attack and sideslip as feedback signals have arisen in the NDI controller due to their appearance as states in the equations of motion. These signals require a calibrated and fail safe airdata system. However, it is possible to replace both of these signals by the nearly equivalent signals of normal acceleration and lateral acceleration respectively.

$$
\begin{aligned}
& n_{z}=n_{\alpha} \cdot \alpha \\
& n_{y}=n_{\beta} \cdot \beta
\end{aligned}
$$

One may also not close the outermost sideslip feedback loop in Fig. 3, thereby ending up with the more traditional controller in the lateral-directional axis $[8,17]$.

The structure we have derived also allows for an angle-ofattack or normal acceleration demand system to be implemented.

\section{SimUlation RESUlTS AND CONCLUSIONS}

To demonstrate the ability of the NDI based controller to follow a typical trajectory: namely an autolanding scenario consisting of level flight segments, level turns, descent profiles and the flare maneuver (Fig. 1). The six-degree-of-freedom simulation result is shown in Figs. 4-6. Longitudinal variables are shown in Fig. 4, while lateral-directional variables are seen in Fig. 5. Finally, in Fig. 6 we see that the actuator rates are well within the maximum values.

In conclusion we have demonstrated a control system design approach based on NDI with the following benefits:

- Control and state decoupling of the innermost loops is achieved

- The procedure for obtaining outer loop design gains is established without compromising the separation of time scales required for the cascaded controller

- Trajectory following design is achieved for the class of fixed wing aircraft including unmanned aerial vehicles

- Alternate control structures which do not depend on airdata signals like angle of attack and sideslip can be derived from this approach. In this manner the NDI controller can be used for maneuvering

\section{REFERENCES}

[1] G. Meyer, R. Su, and L. R. Hunt, "Applications of nonlinear transformations to automatic flight control", Automatica, vol. 20, no. 1, 1984, pp. 103-107.

[2] S. H. Lane, and R. F. Stengel, "Flight control design using nonlinear inverse dynamics", Automatica, vol. 24, no. 4, 1988, pp. 471-483. 
[3] P. K. A. Menon, M. E. Badgett, R. A. Walker, and E. L. Duke, "Nonlinear flight test trajectory controllers for aircraft", Journal of Guidance, Control, and Dynamics, Vol. 10., No. 1, Jan.-Feb. 1987, pp. 67-72.

[4] I. Kanellakopoulos, P. V. Kokotovic, and A. S. Morse, "A toolkit for nonlinear feedback design", Systems \& Control Letters, 18(2):83-92, Feb. 1992.

[5] D. J. Bugajski, and D. F. Enns, "Nonlinear control law with application to high angle of attack", Journal of Guidance, Control, and Dynamics, Vol. 15, No. 3, May-June 1992, pp. 761-767.

[6] S. A. Snell, D. F. Enns, and W. L. Garrard Jr., "Nonlinear inversion flight control for a supermaneuverable aircraft", Journal of Guidance, Control, and Dynamics, Vol. 15, No. 4, July-Aug. 1992, pp. 976-984.

[7] J. S. Brinker, and K. Wise, "Stability and flying qualities robustness of a dynamic inversion aircraft control law", Journal of Guidance, Control, and Dynamics, Vol. 19, No. 6, Nov.-Dec. 1996, pp. 1270-1277.

[8] W. C. Reigelsperger, S. S. Banda, and D. P. Lemaster, "Application of multivariable control theory to aircraft control laws. Final ReportMultivarible control design guidelines", WL-TR-96-3099, Flight Dynamics Directorate, Wright Laboratory, Wright-Patterson AFB, OH, May 1996.

[9] C. J. Miller, "Nonlinear dynamic inversion baseline control law: Flighttest results for the full-scale advanced systems testbed F/A-18 airplane", AIAA Paper no. 2011-6468, AIAA Guidance, Navigaion, and Control Conference, Portland Oregon, 2011.
[10] O. Harkegard, "Backstepping and control allocation with applications to flight control", Ph.D. Thesis, Department of Electrical Engineering Linkoping, Sweden: Linkoping University, 2003.

[11] E. R. Van Oort, "Adaptive backstepping control and safety analysis for modern fighter aircraft", Ph.D. Thesis, Faculty of Aerospace Engineering TU Delft, Netherlands: Delft University of Technology, 2011.

[12] S. Sieberling, Q. P. Chu, and J. A. Mulder, "Robust flight control using incremental nonlinear dynamic inversion and angular acceleration prediction", Journal of Guidance, Control, and Dynamics, Vol. 33, No. 6, Nov.-Dec. 2010, pp. 1732-1742.

[13] M. W. Oppenheimer, D. B. Doman, and M. A. Bolender, "Control allocation for over-actuated systems", in Proceedings of the $14^{\text {th }}$ Mediterranean Conference on Control and Automation (MED '06),pp. 16 , June 2006.

[14] L. T. Nguyen, M. E. Ogburn, W. P. Gilbert, K. S. Kibler, P. W. Brown, and P. L. Deal, "Simulator study of stall/post-stall characteristics of a fighter airplane with relaxed longitudinal static stability," NASA TP1538, 1979.

[15] S. G. Teo, "Autolanding system study: Aerodynamic data of an aircraft with independent control surfaces by CFD," Technical Report of DSO National Lab., 2003.

[16] A. Miele, Flight Mechanics Volume 1: Theory of Flight Paths, AddisonWesley Publishing Company, Inc., 1962.

[17] J. H. Blakelock, Automatic Control of Aircraft and Missiles, John Wiley \& Sons Inc., 1991.

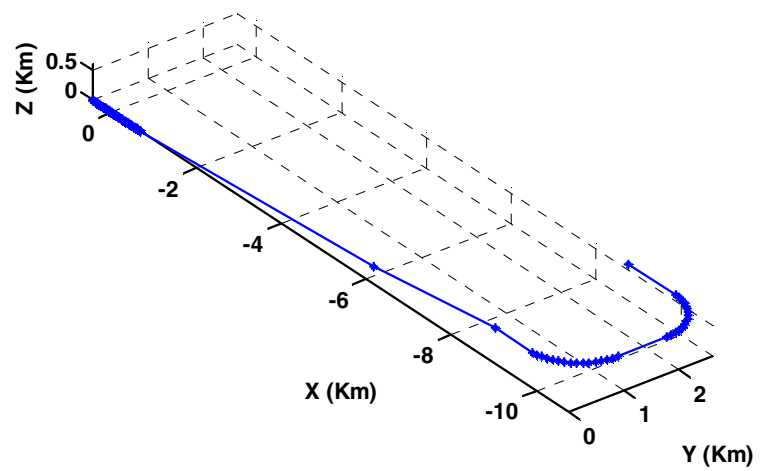

Fig. 1 Autolanding trajectory

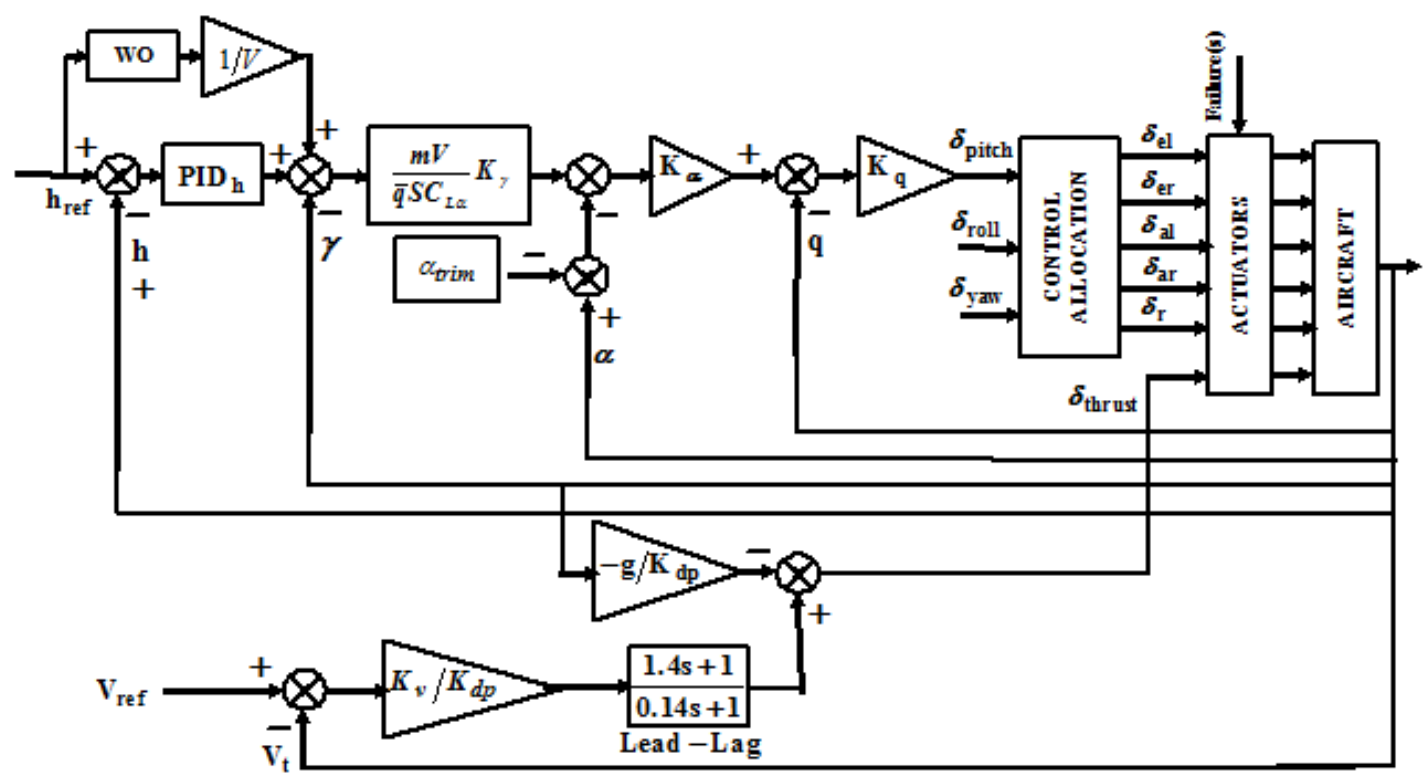

Fig. 2 Longitudinal Axis NDI Controller 


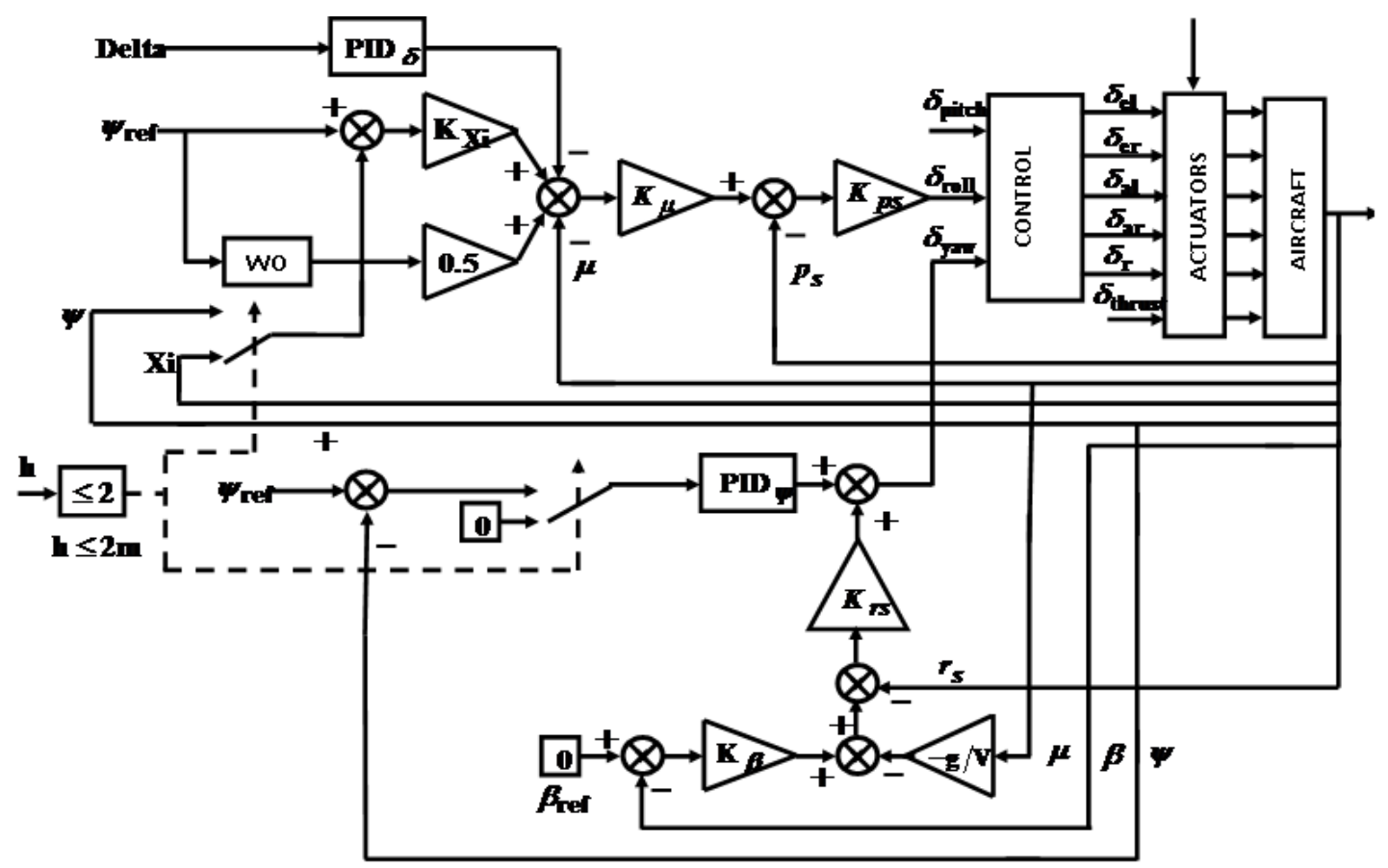

Fig. 3 Lateral-Directional Axis NDI Controller
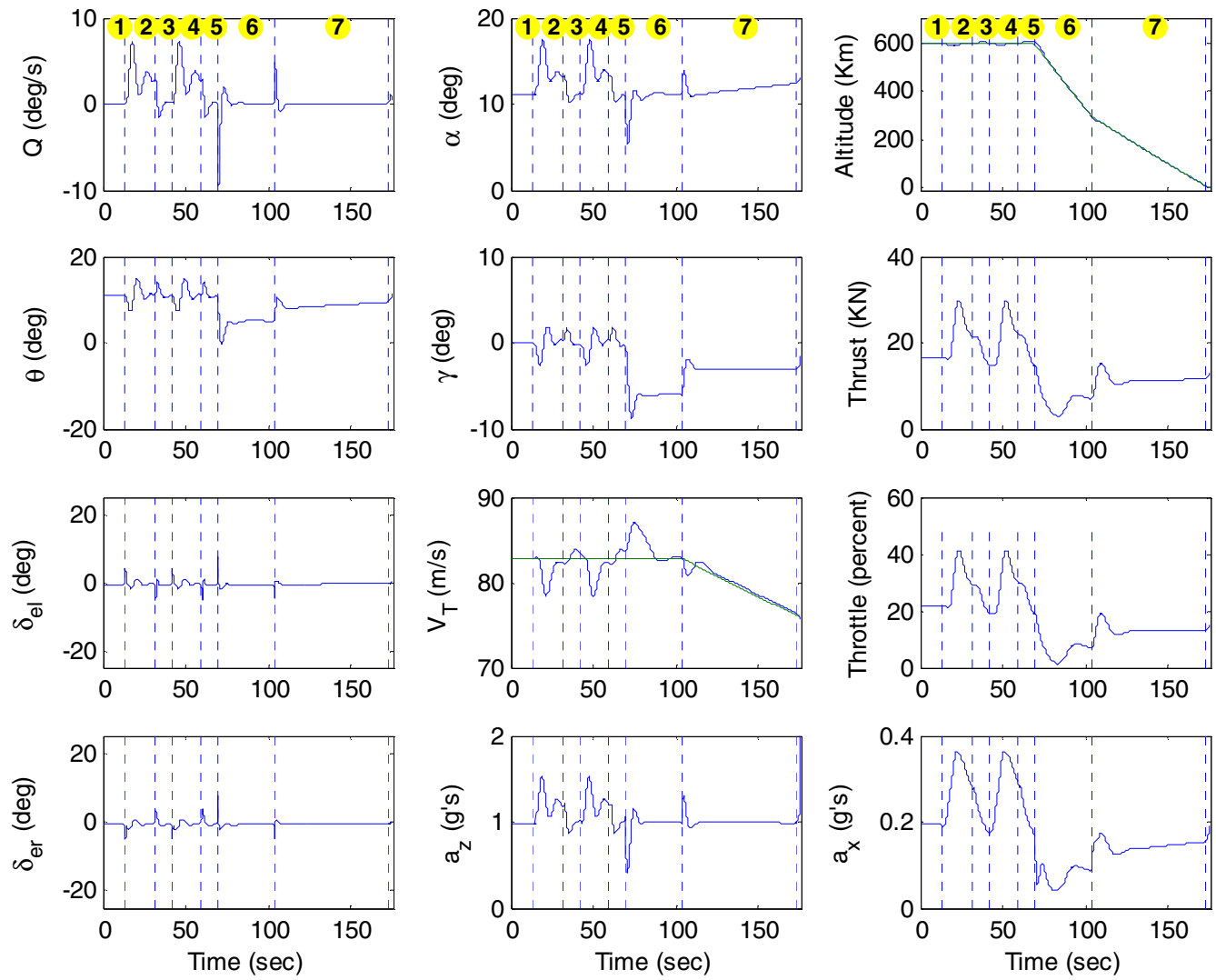

Fig. 4 Longitudinal response during trajectory following 

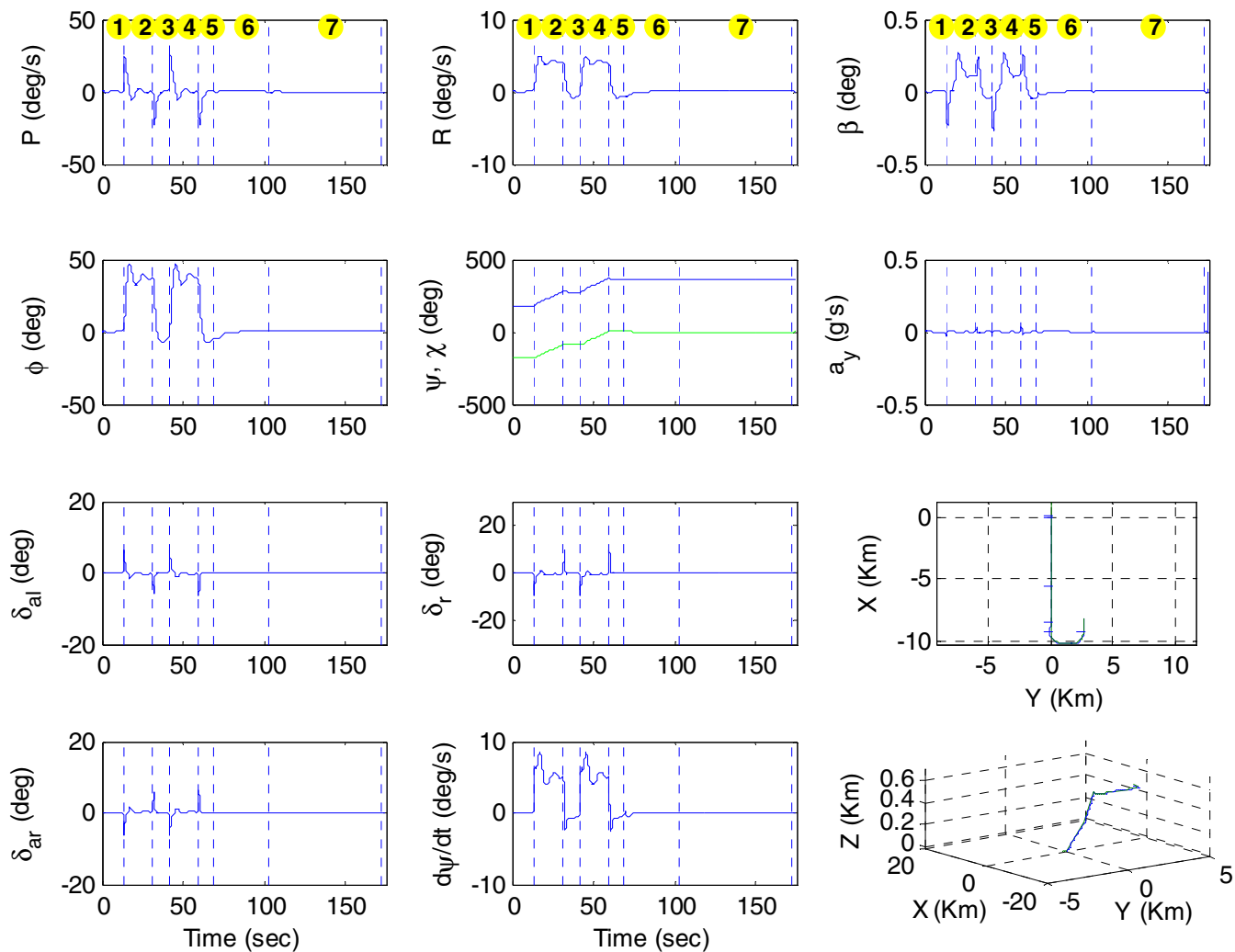

Fig. 5 Lateral-directional response during trajectory following
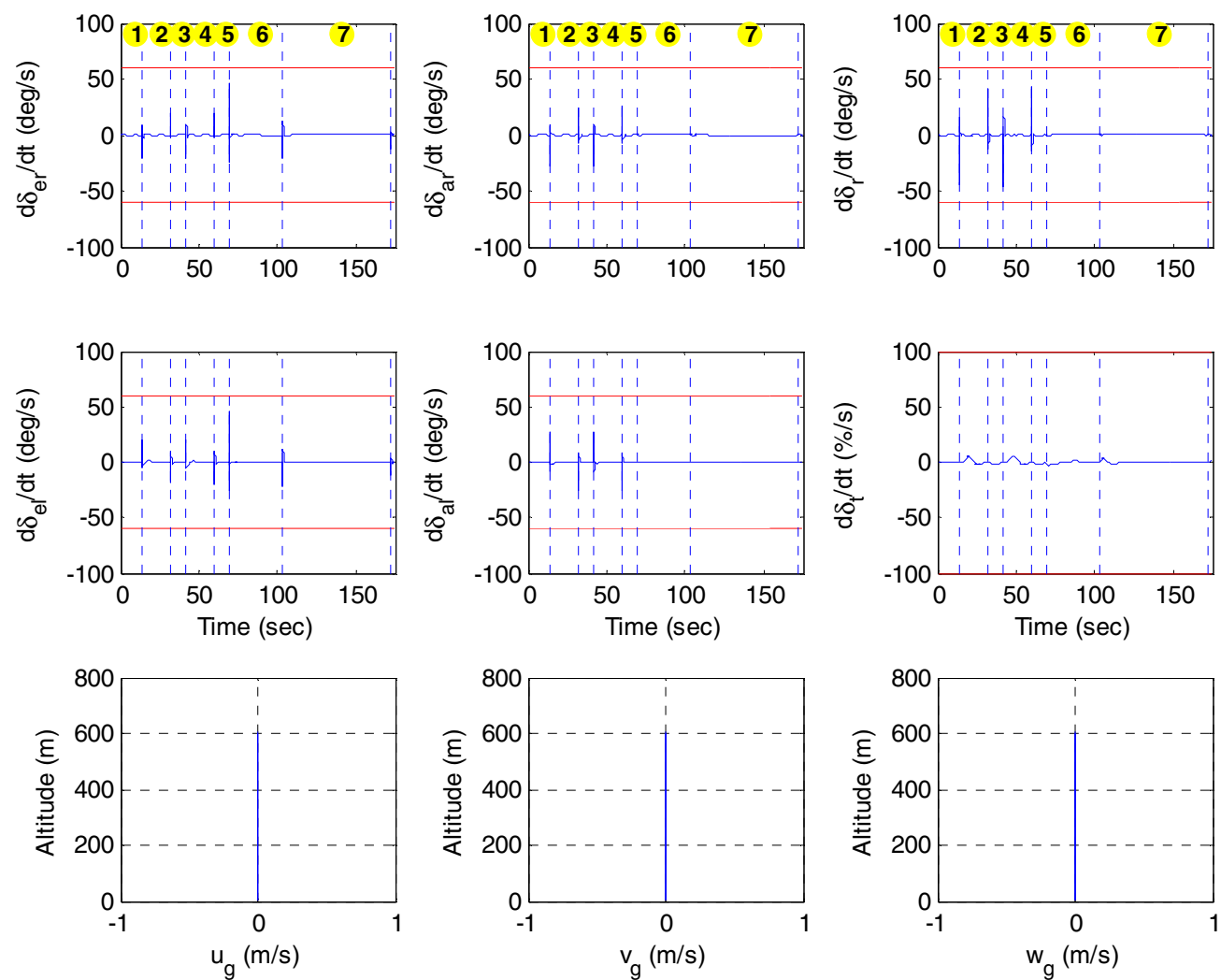

Fig. 6 Actuator rates as function of time and wind profile as function of altitude 